\title{
NUEVOS REGISTROS DE THYSANOPTERA (INSECTA) PROCEDENTES DEL VALLE DE CAÑTE
}

\author{
María E. Ogusuku ${ }^{1}$ \\ Menandro S. Ortiz ${ }^{2}$ \\ Instituto de Medicina Tropical von Humbold \\ Universidad Peruana Cayetano Heredia. ${ }^{1}$ \\ Facultad de Ciencias Biológica \\ Universidad Ricardo Palma. ${ }^{2}$
}

\section{RESUMEN}

Este trabajo fue hecho en base a colectas realizadas en la Provincia de Cañete, $148 \mathrm{~km}$ al sur de Lima, en el Departamento de Lima y tuvo por objetivo ampliar nuestros conocimientos sobre la diversidad de los insectos del Orden Thysanoptera de la zona.

Se logró determinar 8 especies en total, las que pertenecen a 7 géneros diferentes, éstas son: Anaphothrips sudanensis Trybom, Bregmatothrips venustus Hood, Chirothrips frontalis Williams, Chirothrips mexicanus Crawford, Kurtomathrips morrilli Moulton, Plesiothrips perplexus (Beach), Sericothrips annulipes Hood, Thrips tabaci Lindemann.

Todas las especies mencionadas, excepto Thrips tabaci son nuevos para la zona; nuevos registros para el país lo constituyen el género Kurtomathrips Moulton con la especie K. morrilli Moulton,y las especies: Anaphothrips sudanensis Trybom, Chirothrips mexicanus Crawford, Plesiothrips perplexus (Beach) y Sericothrips annulipes Hood.

Palabras Claves: Thysanoptera, trípidos, trips

\section{SUMMARY}

In the present work with Thysanoptera from Cañete's Valley, are identified the following species: Anaphothrips sudanensis Trybom, Bregmatothrips venustus Hood, Chirothrips frontalis Williams, Chirothrips mexicanus Crawford, Kurtomathrips morrilli Moulton, Plesiothrips perplexus (Beach), Sericothrips annulipes Hood, and Thrips tabaci Lindemann.

All the species, except Thrips tabaci Lindemann, are new record to Cañete Valley, but the species Anaphothrips sudanensis Trybom, Chirothrips mexicanus Crawford, Kurtomathrips morrilli Moulton, Plesiothrips perplexus (Beach), and Sericothrips annulipes Hood are new record to Peru.

Key Words: Thysanoptera, tripids, trips

\section{INTRODUCCION}

Los insectos que pertenecen al orden Thysanoptera, se caracterizan por su tamaño diminuto alcanzado en promedio $1.5 \mathrm{~mm}$ de longitud total, por ello generalmente no son percibidos y por lo mismo son muy poco conocidos.

Comúnmente se les conoce con el nombre de "trips" y su distribución es amplia en todos los continentes, principalmente en zonas tropicales y subtropicales, en donde la diversidad de especies es mayor. Algunas especies son altamente polífagas, pero son pocas las consideradas de importancia económica en nuestro medio como Thrips tabaci Lindemann conocido también como el "trips de la cebolla" porque constituye plaga de este cultivo, Leucothrips theobromae que ataca cultivos de cacao, Frankliniella williamsi Hood y $F$. gossypiana Hood en plantas jóvenes de maíz y algodonero, respectivamente; sin 
embargo en otras latitudes se han reportado muchas especies de trips como plagas en plantaciones de importancia agrícola y ornamental, así como vectores de virus fitopatógenos (Lewis, 1973; Palmer et al., 1989).

Desde que Hood (1915) hiciera los primeros registros de trips procedentes de Lima, se han hecho muchos reportes y descripciones de especies nuevas de diferentes zonas, especialmente de la región de selva alta y sierra, siendo las mayores contribuciones de Moulton, Hood y Ortiz y que no necesariamente eran consideradas como de interés agrícola; sin embargo para Cañete, zona de interés de este estudio sólo hay un trabajo que reporta especies correspondientes a este grupo (Ogusuku \& Ortiz, 1991) que reporta 9 especies pertenecientes al género Frankliniella Karny.

Es por esta razón que se llevó a cabo este estudio taxonómico, con la finalidad de incrementar nuestros conocimientos sobre la diversidad de este grupo de insectos en el país y su distribución en la zona de estudio.

\section{ANTECEDENTES}

En el Perú, los primeros estudios de estos insectos fueron hechos por Hood (1915), quien reportó especies nuevas procedentes de Lima, pertenecientes a los géneros Frankliniella Karny y Liothrips Uzel. Posteriormente registró otras nuevas especies de diferentes lugares del país, en su mayor parte, provenientes de la zona de selva alta. Wille (1952) citó por primera vez algunas especies de importancia económica y sus métodos de control. Soukup (1944), hizo una recopilación de todos los Thysanoptera registrados para nuestro medio, totalizando hasta aquella época, 40 géneros y 104 especies.

Otros trabajos sobre Thysanoptera del Perú pertencen a Stannard (1972), quien describe dos especies nuevas colectadas en Machu Picchu, Cusco; Ortiz (1972, 1973 a, b, c) y a Ogusuku \& Ortiz (1991), sumando un total de 135 especies, repartidos en 52 géneros entre terebrantios y tubulíferos.
En nuestro medio, de los 52 géneros registrados, 14 corresponden a la familia Thripidae, 33 pertenecen a los Phlaeothripidae y los 5 restantes corresponden a las otras familias de los Terebrantia (Ortiz, 1972, 1973a,b; Soukup, 1944).

\section{MATERIAL Y METODOS}

\section{Zona de estudio}

El Valle del río Cañete se ubica políticamente en la Provincia de Cañete, $149 \mathrm{~km}$ al sur de Lima, capital del Departamento del mismo nombre. Geográficamente la cuenca del río Cañete se sitúa en la región central y occidental del Perú y el Valle se encuentra íntegramente en la çosta central.

Ecológicamente la zona del valle se define como Desierto Sub-Tropical (ONERN, 1970) y se extiende desde el litoral hasta los $2000 \mathrm{msnm}$. Presenta un clima dominante de tipo muy seco y semi-cálido, con temperatura promedio de $19.7^{\circ} \mathrm{C}$ siendo la precipitación promedio anual de las partes bajas de $27.9 \mathrm{~mm}$ y en las zonas altas de hasta $200 \mathrm{~mm}$ anuales, con una Humedad Relativa promedio de $81 \%$ en la zona baja y $84 \%$ en la zona alta.

\section{Colección de las muestras}

El material biológico para este trabajo fue colectado directamente durante los meses de Enero, Febrero y Marzo de 1986 principalmente en las zonas ribereñas del Río Cañete. Esta área agrícola es intensamente asperjado con insecticidas de origen químico; haciendo que la fauna entomológica de interés sea mínima según se comprobó.

Las colectas se realizaron en diferentes localidades de la Provincia, ubicadas en los Distritos de Cerro Azul, San Luis, San Vicente de Cañete, Imperial, Quilmaná, Nuevo Imperial, Lunahuaná, Pacarán y Zúñiga. 
El material entomológico colectado en el campo fue llevado al Laboratorio de Entomología de la Universidad Nacional Agraria La Molina, en Lima para su procesamiento e identificación taxonómica, previo montaje en láminas de microscopía.

\section{RESULTADOS}

\section{Género Anaphothrips Uzel}

Este género se caracteriza porque carece de setas largas, especialmente sobre el pronotum.

Anaphothrips sudanensis Trybom, 1911

Euthrips flavicintus Karny, 1912

Anaphothrips bicolor Morgan, 1925

Anaphothrips (Odontoanaphothrips) enceliae Moulton, 1926

Anaphothrips flavicintus Bailey, 1948.

Esta especie constituye un nuevo registro en nuestro país y, al igual que otras especies de este género, aparentemente tiene preferencia por las gramíneas como hospederos según lo reporta Medina Gaud (1961), Stannard (1968), Ortiz (1973) y Palmer et al. (1989).

Material Examinado: Quilmaná: 2 hembras en cogollo de Sorghum halepense (4.II.86)

Distribción geográfica: Chipre, Palestina, Sudan, Marruecos, Egipto, Somalia, Mozambique, Sudáfrica, Uzbequistán, Asia Central, Formosa, India, Ceylan, Java, Sumatra, Australia, Nueva Bretaña, Nueva Caledonia, Puerto Rico, Cuba (Jacot Guillarmod, 1974).

\section{Género Bregmatothrips Hood}

Como característica de importancia éste género presenta en el pronotum 2 pares de setas postero-angulares y un par de setas antero-marginales largas, estas últimas algo distanciadas del margen externo. Los machos son siempre branquípteros.

\section{Bregmatothrips venustus Hood}

Bregmatothrips venustus Hood, 1912
Esta especie fue registrada por primera vez en nuestro país por Ortiz (1973b) para la zona de Lima. Los ejemplares colectados por Ortiz como los que aquí se presentan tienen sólo 6 segmentos antenales, aún cuando Stannard (1968) registra para Illinois (USA), la misma especie pero con 8 segmentos antenales. Esta diferencia se debe a que nuestros especímenes presentan los últimos segmentos fusionados, constituyendo el VI segmento antenal.

Material Examinado: Quilmaná: 17 hembras y 5 machos en Eleusine indica (7.I.86); 6 hembras y 1 macho en Sorghum halepense (4.II.86).

Distribución Geográfica: Hawaii, Cuba, México, USA (Bailey, 1957); Argentina (De Santis, 1964); Perú (Ortiz, 1973b).

\section{Género Chirothrips Haliday}

Las características sobresalientes para determinar a este género son el pronotum trapezoidal con 2 pares de setas posteroangulares relativamente largas.

\section{Chirothrips frontalis Williams}

Chirothrips frontalis Williams, 1914

Chirothrips sulcatus John, 1922.

Las características de los especímenes colectados en este trabajo, son prácticamente idénticas a los reportados por Andre $(1939,1941)$.

Material Examinado: Imperial: 9 hembras y 3 machos en espigas de Sorghum halepense (7.I.86).

Distribución Geográfica: Australia (Mound \& Palmer, 1972); USA (Andre, 1939, 1941); Argentina, Sudáfrica (Andre, 1941), Perú (Ortiz, 1973).

\section{Chirothrips mexicanus Crawford}

Chirothrips mexicanus Crawford,1909 Chirothrips mexicanus Watson, 1920 Chirothrips floridensis var. catchingsi Watson, 1924. 
Esta especie constituye un nuevo registro para nuestro país. Las características que presentan nuestros ejemplares son muy similares a las que describen Medina Gaud (1961) para ejemplares de Puerto Rico y Stannard (1968) para aquellos procedentes de Illinois (USA); aunque también éste autor hace notar que en el metasternum lleva un grupo de 50 setas o más, gruesas y cortas, y en nuestros ejemplares ninguno llega a tener más de 46 setas cortas.

Material Examinado: Imperial: 6 hembras en Eleusine indica (7.I.86).

Distribución geográfica: USA hasta Panamá (Hood, 1939); Australia (Mound \& Palmer, 1972); Puerto Rico (Medina Gaud, 1961); Brasil (Moulton, 1938); Filipinas, Indias Occidentales, Sudamérica y Hawaii (Andre, 1939).

\section{Género Kurtomathrips Moulton}

Todo el cuerpo totalmente ornamentado con sinuosos engrosamientos. Todas las setas son anchas y curvadas.

\section{Kurtomathrips morrilli Moulton}

Kurtomathrips morrilli Moulton, 1927

El género y la especie constituyen un nuevos registros en nuestro país. Todos los registros anteriores se limitan al hemisferio norte. El ejemplar observado presenta las mismas características que Moulton (1927) describiera en su reporte original, excepto por las características de las setas de los segmentos IX y X que Moulton considera rectos, pero nuestro ejemplar los tienen curvos; Bailey (1957) hace notar que las setas son anchas, curvadas y algo dentada en el ápice, siendo este último detalle una característica que no presenta nuestro ejemplar e igualmente este autor describe para la especie morrilli una hilera continua de peine cuyos dientes están esparcidos y abruptamente redondeados, refiriéndose a las esculturas en forma de festones que presenta cada segmento abdominal I al VIII y que Moulton en su descripción original considera como aserrado.
Material Examinado: Pacarán: 1 hembra en flores de Coniza floribunda (26.III.86).

Distribución geográfica: USA (Moulton, 1927); Hawaii (Sakimura, 1956); Jamaica (Bailey, 1961).

\section{Género Plesiothrips Hood}

Tienen 2 setas postero-angulares largas sobre el pronotum y las hembras tienen el ovipositor débilmente desarrollado.

\section{Plesiothrips perplexus (Beach)}

Sericothrips perplexa Beach, 1896.

Plesiothrips perplexus Hood, 1915.

El registro de Plesiothrips perplexus es nuevo para nuestro país. Sus características morfológicas son muy similares a las que reportan Medina Gaud (1961) para ejemplares de Puerto Rico.

Material Examinado: Imperial: 8 hembras colectadas de hojas jóvenes de Sorghum halepense (4.II.86).

Distribución geográfica: USA (Stannard, 1968); Puerto Rico (Medina Gaud, 1961).

\section{Sericothrips Haliday}

Rhytidothrips Karny, 1910

Hydatothrips Karny, 1913.

Una característica que distingue a este género son las marcadas estriaciones que presenta en la cabeza y el pronotum, y las manchas ligeramente más oscuras de los apodemas sobre el pronotum, muy característicos; y también por las densas microsetas que preesntan sobre los segmentos abdominales.

\section{Sericothrips annulipes Hood}

Sericothrips annulipes Hood, 1927.

Constituye un nuevo registro para nuestro país. Es muy similar a la descripción que hace Stannard (1968) de esta especie para ejemplares procedentes de llinois (USA), con la diferencia de que su ejemplar no presenta microsetas en la parte media de los 
tergitos abdominales y considera que a veces el peine del segmento VII son débiles hacia el centro del tergito, lo cual no coincide con nuestros ejemplares que cuyas microsetas son muy densas en todos los segmentos abdominales y el peine del tergito VII es uniforme.

Material Examinado: Quilmaná: 2 hembras en flores de Bidens pilosa (7.I.86).

Distribución Geográfica: USA (Stannard, 1968).

\section{Género Thrips Linnaeus}

Este género se caracteriza porque presenta sólo 2 pares de setas postero-angulares y 3 a 4 pares de setas postero-marginales pequeñas. Además de finas estriaciones sobre la cabeza y pronotum.

\section{Thrips tabaci Lindemann}

Thrips tabaci Lindemann, 1888

Thrips minutissima Curtis 1846

Thrips striatus Guillete, 1893

Thrips bicolor Karny, 1907

Thrips bremnerii Moulton, 1907

Thrips dianthi Moulton, 1936

Thrips evestignatus Oettingen, 1947

Especie cosmopolita, comunmente conocida como el "thrips de la cebolla", pués plaga de este cultivo donde puede causar severos daños, siendo además vectora de virus fitopatógenos, lo que incrementa su importancia económica. También es posible hallarla sobre otros hospederos causando problemas, tal como lo señala Stannard (1968).

Material Examinado.- San Luis: 4 hembras en hojas de Allium cepa (18.I.86); Imperial: 7 hembras en flores de Chrysantemun sp (7.I.86); 1 hembra en flores de Dianthus caryophyllus (9.I.86).

Distribución Geográfica: USA (Stannard, 1968; Gentile \& Bailey, 1968); Puerto Rico (Medina Gaud, 1961), Perú (Soukup, 1944).

\section{DISCUSIÓN}

En el país pocos son los investigadores que han contribuido al conocimiento de estos insectos. Se puede citar a Hood (1915) como el investigador que iniciara los trabajos sobre Thysanoptera, quien describió muchas especies como aquellas que pertenecen al género Frankliniella Karny, las mismas que fueran nuevamente registradas por Ortiz $(1972,1973)$.

En el presente trabajo ha sido posible incrementar el número de registros para nuestro país como son Kurtomathrips morrilli Moulton, Anaphothrips sudamensis Trybom, Chirothrips mexicanus Crawford, Plesiothrips perplexus (Beach) y Sericothrips annulipes Hood.

En este sentido conssideramos posible la presencia de estas especies en nuestro medio dado que de igual manera se reporta para otros países que integran la región neotropical, como se logra apreciar en el catálogo elaborado por Jacot-Guillarmod (1970 - 1974). Los hospederos de igual manera los observamos como buenos indicadores, en concordancia con los diferentes trabajos revisados.

\section{REFERENCIAS BIBLIOGRÁFICAS}

1. ANDRE, F. 1939. A synopsis of the american species of Chirothrips Haliday (Thysanoptera). Proc. Ent. Soc. Wash., 41(6): 192-204.

2. ANDRE, F. 1941. Two new species of Chirothrips Haliday with notes on Chirothrips frontalis Williams (Thysanoptera: Thripidae) Ann. Ent. Soc. Am., 34 (2): 451-457.

3. BAILEY, S.F. 1957. The Thrips of California. Part I: Suborder Terebrantia. Bull. Calif. Ins. Survey 4 (5): 143-220.

4. BAILEY, S.F. 1961. A review of the genus Kurtomathrips with the descriptiuon of a new species. Proc. Ent. Soc. Wash. 63 (4): 257-260.

5. DE SANTIS, L. 1964. Tisanópteros de la Provincia de La Rioja (República Argentina). Rev. Mus. La Plata Zool 8 (58): $39-46$ 
6. GENTILE, A.; BAILEY. S.F 1968. A revision of the genus Thrips Linnaeus in the New World with a catalogue of the world species (Thysanoptera: Thripidae). Univ. Cal. Publ. Ent. 51:193.

7. HOOD, J.D. 1915. An outline of the subfamilies and higher groups of the insect Order Thysanoptera. Proc. Biol. Soc. Wash. 28: 53-60.

8. HOOD, J.D. 1939. Notes on Chirothrips, witth descriptions of two new species (Thysanoptera). Rev. Ent. (Rio Jan.) 10 (2): 461-471.

9. JACOT-GUILLARMOD, C.F. 19701974. Catalogue of the Thysanoptera of the world ) Parts 1-4, Terebrantia). Ann. Cape Prov. Mus. (Nat. Hist.).

10. LEWIS, T.R. 1973. Thrips: Their Biology, Ecology and Economic Importance, N.Y. Ac. Press., 349 pp.

11. MEDINA-GAUD, S. 1961. The Thysanoptera of Puerto Rico. Agr. Exp. Sta., P. Rico, Tech. Paper 32, 159 pp.

12. MOULTON, D. 1927. New species and notes. Bull. Brook. Ent. Soc. 22 (4): $187-190$

13. MOULTON, D. 1938. Thysanoptera from Minas Gerais (Brazil). Rev. Ent. (Rio Jan.),1(1-2): 314-322.

14. MOUND, L.A. ; PALMER; J.M. 1972. Grass-flower infesting thrips of the genus Chirothrips Haliday in Australia. Jour. Austr. Ent. Soc. 11: 332-339.

15. ONERN. 1970. Inventario, Evaluación y uso Racional de los Recursos Naturales de la Costa. Cuenca del Rio Cañete. Vol. I y II.

16. OGUSUKU, M.E.; ORTIZ, M.S. 1991. Nueve especies del género
Frankliniella Karny (Thysanoptera: Thripidae) procedentes de Cañete. Rev. Per. Ent. 33: 125.

17. ORTIZ, M.S. 1972 Contribución al conocimiento de los Thysanoptera de Lima. Rev. Per. Ent.15 (1): 83-91.

18. ORTIZ, M.S. 1973a. Contribución al conocimiento de los Thysanoptera del Perú. Rev. Per. Ent. 16 (1): 111-114.

19. ORTIZ, M.S. 1973b . Dos nuevos registros de Thysanoptera para el Perú. Rev. Per. Ent. 16 (1): 115-117.

20. ORTIZ, M.S. 1973c. Una nueva especie de Isoneurothrips Bagnall (Thysanoptera: Thripidae) del Perú. Rev. Per. Ent. 16 (1): 117-120.

21. PALMER, J.M.; MOUND, L.A; DU HEALIME, G.J. 1989. 2. Thysanopotera. CIE Guides to Insects of Importance to Man. CAB International Inst.Ent., British Mus., Nat. Hist., 73 pp.

22. SAKIMURA, K. 1956. Kurtomathrips morrilli a non-vector of the spotted wilt virus, with notes on Liothrps urichi. Jour. Econ. Ent. 49 (4): 562.

23. SOUKUP, J. 1944. Los Tisanópteros Peruanos. Bol. Mus. Hist. Nat. J. Prado, 28-29: 57-66.

24. STANNARD, L.J. 1968. The Thrips or Thysanoptera of Illinois. Illinois Nat. Hist. Surv. Bull. 29 (4): 215-552.

25. STANNARD, L.J. 1972. Three new species and one new genus of Heterothripidae (Thysanoptera) from South America. Proc. Biol. Soc. Wash. 84 (41): $335-343$.

26. WILLE, J.E. 1952. Entomología Agrícola del Perú. Junta de Sanidad Vegetal, Ministerio de Agricultura, Segunda Edición, 543 pp. 